\title{
Attentes des industriels en matière d'analyse des oléagineux et des corps gras
}

Oléagineux, Corps Gras, Lipides. Volume 10, Numéro 4, 242-7, JUILLET-AOÛT 2003, Innovations dans I'analyse et le traitement des données

Auteur(s) : Pierre-Yves VIGNERON, LESIEUR R\& -- Coudekerque-Branche .

\section{ARTICLE}

Auteur(s) : Pierre-Yves VIGNERON

LESIEUR R\&D - Coudekerque-Branche

Toute production industrielle, quelle qu'elle soit, nécessite un minimum de contrôles. L'industrie des corps gras n'échappe pas à cette règle. Les contrôles sont nécessaires :

- Avant le démarrage d'une chaîne de fabrication, pour analyser les matières premières qui vont être mises en œuvre et les réactifs qui seront utilisés.

- Lors du démarrage de certaines machines, pour vérifier la régularité de leur fonctionnement.

- Au cours du process, pour pallier à toute dérive de la ligne et pour vérifier si les spécifications préalablement définies sont respectées en certains points cruciaux de la chaîne et sur le produit fini avant stockage ou cession à la clientèle. La maîtrise d'une production nécessite le contrôle de paramètres physiques et physico-chimiques. Les premiers sont suivis à l'aide de capteurs généralement livrés avec le matériel de production. Ce sont des mesures de température, pression, débit, niveau... Pour assurer le contrôle des paramètres physico-chimiques, différentes organisations sont possibles (figure 1).

Laboratoire de contrôle
Cellules de contrôle d'atelier
Tests physico-chimiques
Capteurs, automates

Figure 1.

- Le laboratoire de contrôle est généralement un service indépendant de la production, qui analyse selon des procédures parfaitement définies et à une fréquence préétablie, des échantillons prélevés en différents endroits de la chaîne. II présente l'avantage de suivre une méthodologie rigoureuse, mais une telle organisation manque souvent de souplesse et les délais de réponse sont trop importants pour des lignes de production à haut débit. 
- La cellule de contrôle d'atelier est située dans l'atelier sous la responsabilité du chef de service ; elle permet de suivre les paramètres essentiels à une cadence variable selon les situations; par exemple très élevée si des anomalies apparaissent ou lors des démarrages de la ligne de production, ou réduite en marche normale. Une telle structure a l'avantage de la souplesse et de la réactivité mais peut poser des problèmes de fiabilité, étant juge et partie.

- Les tests physico-chimiques. réalisés par les opérateurs sur la ligne présentent l'avantage de leur donner la possibilité d'agir immédiatement mais de tels outils manquent souvent de précision.

- Les capteurs et automates sont des équipements qui donnent l'information en temps réel ou différé, rendant ainsi possible l'automisation d'une ligne. Cependant ils nécessitent un entretien soigné pour rester fiables et sont rarement disponibles commercialement. La tendance d'avoir désormais des lignes de production de grande capacité oblige à s'orienter vers des contrôles par des capteurs ou des tests physico-chimiques afin de pouvoir réagir très rapidement. Les contrôles des matières premières à réception et des produits finis avant stockage ou livraison sont indispensables. Pour les matières premières, il s'agit de vérifier que leurs caractéristiques physico-chimiques correspondent aux spécifications attendues. En effet les résultats analytiques vont conditionner leur prix d'achat, les modalités d'un éventuel stockage, l'adoption d'un traitement spécifique si nécessaire, les pertes de rendement, la réduction des cadences et bien sûr la qualité du produit fini. Si l'usine n'a pas la possibilité de différer ces contrôles par suite d'un manque de stockage ou d'un fonctionnement en flux tendu, les déterminations devront être rapides et précises pour éviter toute attente au déchargement. Pour les produits finis, il est nécessaire de s'assurer qu'ils sont conformes aux spécifications officielles et répondent aux critères de qualité définis par l'entreprise d'autant plus si ce sont des produits de marque. Dans les deux cas - matières premières et produits finis - les contrôles à effectuer font souvent appel à des méthodes physico-chimiques qui ne peuvent être réalisées qu'en laboratoire. Pour illustrer ces différents aspects du problème, nous avons listé les moyens de contrôle à utiliser pour la fabrication des huiles brutes, la fabrication des huiles raffinées et mis en avant ceux qui paraissent les plus appropriés pour qu'un atelier maîtrise au mieux la qualité de sa production.

\section{Production des huiles brutes}

Dans le cas de la production des huiles brutes de graines, il ressort que les paramètres à contrôler le plus fréquemment en cours de process sont (figure 2) : Les teneurs :

- en matières grasses, en particulier des tourteaux (taux de déshuilage),

- en humidité

- en solvant résiduel.

Il reste à ajouter les analyses destinées à contrôler la qualité des graines à triturer et des tourteaux avant expédition. Il faut aussi prévoir désormais dans certains cas le contrôle de l'absence de graines OGM.

L'industriel a à sa disposition des méthodes officielles qui ont fait l'objet de normalisation de la part d'organismes nationaux ou internationaux. Ces méthodes sont généralement peu adaptées à une utilisation de routine en atelier, car le délai de réponse est trop long et/ou l'équipement inapproprié compte tenu du personnel disponible et de sa qualification. L'industriel va faire appel à des 
techniques d'analyse plus rapides ou recherchera des capteurs ou des automates, la rapidité d'information prenant le dessus sur la sensibilité de la mesure. Nous avons listé les méthodes qui peuvent être appliquées pour assurer ces contrôles (figure 3).

\begin{tabular}{|c|c|c|c|}
\hline $\begin{array}{l}\text { Paramètres } \\
\text { contrôler }\end{array}$ & à Méthode normalisée & Autres possibilités & $\begin{array}{l}\text { Capteurs, } \\
\text { Automates }\end{array}$ \\
\hline \multirow{3}{*}{\begin{tabular}{|lr}
$\begin{array}{l}\text { Teneur en matière } \\
\text { grasse }\end{array}$ & (graines - \\
tourteaux) &
\end{tabular}} & \multirow{3}{*}{ Soxhlet RMN } & Densité d'un miscella & / \\
\hline & & Appareils commerciaux & / \\
\hline & & Réflexion dans proche infrarouge & X \\
\hline \multirow[t]{4}{*}{ Matières volatiles } & Dessiccation à l'étuve & Thermobalance & / \\
\hline & & Absorption des micro-ondes & $x$ \\
\hline & & Constante diélectrique & $x$ \\
\hline & & Réflexion dans proche infrarouge & X \\
\hline Résidu de solvant & $\begin{array}{l}\text { Chromatographie } \\
\text { l'espace de tête }\end{array}$ & $\begin{array}{l}\text { Infrarouge de l'espace de tête } \\
\text { Variations sur la technique } \\
\text { chromatographique }\end{array}$ & $\begin{array}{l}\text { / } \\
1\end{array}$ \\
\hline Impuretés & Tamisage et triage & / & / \\
\hline Matières protéiques & Méthode de Kjeldahl & $\begin{array}{l}\text { Appareils commerciaux Réflexion/ } \\
\text { dans proche infrarouge }\end{array}$ & / \\
\hline Cellulose & Méthode de Weende & Appareils commerciaux & / \\
\hline Détection OGM & $\begin{array}{l}\text { Amplification ADN par } \\
\text { PCR }\end{array}$ & Tests immunoenzymatiques & / \\
\hline Glucosinolates & $\begin{array}{l}\text { Chromatographie liquide } \\
\text { Fluorescence aux rayons } \\
\text { X }\end{array}$ & & / \\
\hline
\end{tabular}

Figure 3. Production des huiles brutes.

\section{Matières grasses}

La détermination des teneurs en matières grasses traditionnellement effectuée au Soxhlet pendant plusieurs années bénéficie maintenant de techniques beaucoup plus rapides comme la RMN ou la 
réflexion dans le proche infrarouge. Dans les deux cas, les appareils sont conçus pour être intégrés sur le site de production car leur utilisation ne nécessite pas de personnel spécialisé. Seuls les étalonnages sur lesquels reposent la validité des mesures doivent être réalisés par du personnel compétent.

Les mesures ne prenant que quelques dizaines de secondes, les appareils peuvent analyser un grand nombre d'échantillons dans un minimum de temps permettant ainsi un contrôle statistique de la production.

La méthode RMN est normalisée ISO. Certains appareils différencient les protons de l'eau liée des protons de l'huile à partir de la mesure des temps de relaxation T1 et T2 permettant ainsi de doser simultanément l'eau et l'huile des graines oléagineuses.

Dans le cas où l'échantillon a été broyé, il faut s'assurer que le mode de broyage de l'échantillon introduit dans l'appareil de mesure a été optimisé et reste le même tout au long de l'année, la granulométrie et la perte d'humidité lors des broyages pouvant engendrer des problèmes de reproductibilité.

Ces deux techniques utilisées à proximité de l'atelier donnent aux opérateurs l'information dont ils ont besoin dans un temps réduit. Les autres techniques de détermination de la matière grasse basées sur les mesures de densité d'un miscella obtenu en broyant l'échantillon dans un solvant approprié

ininflammable ne peuvent être utilisées qu'en laboratoire. D'autres appareils commerciaux utilisent des méthodes d'extraction beaucoup plus rapides que le soxhlet et en toute sécurité pour l'utilisateur. Ils sont fort utiles puisque l'on peut utiliser différents solvants d'extraction et poursuivre les analyses sur la matière grasse ainsi extraite.

\section{Matières volatiles}

Les teneurs en matières volatiles sont traditionnellement effectuées au laboratoire par dessiccation dans une étuve. Certains appareils commerciaux appelés thermobalances permettent de réaliser cette opération plus simplement par chauffage infrarouge, réduisant ainsi sensiblement le temps de dessiccation et donnant automatiquement le taux d'humidité. Plus simplement encore, il existe des appareils de laboratoire basés sur la mesure de la réflexion dans le proche infrarouge. De tels matériels sont d'ailleurs homologués pour le contrôle de l'humidité des céréales et peuvent souvent être utilisés sur les échantillons tels quels. Industriellement, il existe des capteurs mesurant en continu la teneur en humidité, certains par absorption d'ondes hyperfréquences, d'autres basés sur le ralentissement des neutrons, ou encore la mesure de la constante diélectrique et bien sûr la réflexion dans le proche infrarouge. Pour la mesure de l'humidité, le responsable d'atelier a ainsi à sa disposition le choix entre plusieurs capteurs qui lui permettent d'optimiser en continu le séchage ou I'humidification des tourteaux.

\section{Résidu de solvant}

La méthode, simple dans son principe, ne peut être mise en œuvre que dans un laboratoire puisqu'elle nécessite un chromatographe en phase gazeuse et de respecter les conditions de génération de l'espace de tête.

II n'y a pas de méthode pratique d'atelier comme il peut en exister pour mesurer la concentration d'hexane dans l'atmosphère. 


\section{Impuretés}

C'est aussi un paramètre pour lequel il n'existe pas de méthode rapide et l'on doit recourir au tamisage et tri manuel, en tenant compte de la taille de l'échantillon pour assurer le maximum de représentativité.

\section{Matières azotées}

La détermination de la teneur en protéines selon la méthode de Kjeldahl est peu commode et dangereuse.

Des appareils commerciaux permettent de réaliser cette analyse en laboratoire tout en respectant les différentes étapes prévues dans le mode opératoire officiel. En les équipant d'un passeur d'échantillons, on peut programmer des séries d'analyses, chacune ne prenant que quelques minutes, et avoir ainsi un fonctionnement quasi automatique en toute sécurité. Suite aux besoins de l'industrie de l'alimentation animale, il existe des équipements fonctionnant sur le principe de la méthode de Dumas et capables de faire des déterminations de l'azote total en quelques minutes. Plus simplement, les appareils de réflexion dans le proche infrarouge sont aussi capables de déterminer la teneur en protéines.

\section{Cellulose}

Ce terme impropre désigne la matière organique qui reste insoluble lorsque l'échantillon subit un traitement acide puis un traitement basique. Cette méthode longue et délicate peut être réalisée de façon moins astreignante avec des appareils commerciaux de laboratoire semi-automatisés conçus pour respecter le protocole d'analyse officiel et capables d'analyser 2 à 6 échantillons simultanément mais aussi par réflexion dans le proche infrarouge.

\section{OGM}

La commercialisation de kits permet à l'industriel d'avoir une information rapide sur les taux de contamination à réception des matières premières. Basés sur des méthodes immunoenzymatiques, ils permettent la détection d'OGM de $0,1 \%$ à $3 \%$ en une demi-journée. La validation des résultats repose sur la représentativité de l'échantillon de graines ou de tourteaux examinés. L'industriel doit malgré tout recourir régulièrement à un laboratoire habitué à ce type de contrôle pour confirmer les résultats, utilisant les techniques d'analyse génétique basées sur la recherche de séquences d'ADN caractéristiques des différentes constructions transgéniques.

\section{Glucosinolates}

Il existe des méthodes normalisées par HPLC pour analyser plusieurs dizaines d'échantillons par jour mais avec un délai de réponse de $48 \mathrm{~h}$.

La fluorescence $X$ est une des techniques rapides qui peut être utilisée pour mesurer les composés soufrés dans le colza. La technique est non destructive, l'identification et la quantification de soufre réalisée en moins d'une minute sur un large domaine de concentration de quelques ppm à quelques pourcents. Les résultats ne sont pas toujours fiables mais la praticité de la technique rend l'investissement intéressant quand on a un grand nombre d'échantillons puisqu'il peut être équipé d'un passeur d'échantillons. 
Lorsque l'on fait le bilan des contrôles à réaliser lors de la production des huiles brutes, on constate que le responsable d'un atelier pression-extraction a à sa disposition plusieurs outils pour mesurer les caractéristiques essentielles des graines et des tourteaux qu'il traite. Certains peuvent être utilisés par du personnel peu qualifié et donnent les résultats avec un délai de réponse très court et suffisant pour un réglage de ligne.

La réflexion dans le proche infrarouge à transformée de Fourier permet de mesurer simultanément plusieurs paramètres essentiels (teneur en matière grasse, humidité, matières azotées, cellulose). II existe de nombreux appareils commerciaux bien adaptés aux mesures sur graines oléagineuses et tourteaux, en version de laboratoire ou de ligne. La qualité des résultats obtenus, leur exploitation nécessitent que les calibrations pour les différents paramètres mesurés aient été réalisées avec soin à partir d'un grand nombre d'échantillons produits sur le site et couvrant les domaines de valeurs souhaitées.

D'autres critères physico-chimiques ne peuvent être mesurés qu'en laboratoire, car ils nécessitent des équipements spécifiques. II y a donc une attente des industriels pour avoir dans ces cas particuliers, par exemple la détection des OGM, la recherche des impuretés dans les graines, la détermination des teneurs en hexane dans les tourteaux au niveau des toasters..., des moyens de mesures plus rapides.

\section{Production des huiles raffinées}

Le responsable d'un atelier de raffinage doit disposer de données analytiques concernant les huiles mises en œuvre, données indispensables aux opérateurs pour adapter leur procédure de raffinage afin d'avoir les meilleurs rendements tout en respectant les spécifications. Les figures 4 et 5 rassemblent les critères à mesurer aux différentes étapes du raffinage depuis I'huile brute jusqu'à l'huile conditionnée ainsi que les méthodes de contrôle disponibles.

\begin{tabular}{|c|c|c|c|}
\hline \multicolumn{2}{|c|}{ Résidu de solvant } & \multicolumn{2}{|c|}{ Sécurité des opérateurs } \\
\hline \multirow{3}{*}{$\begin{array}{l}\text { Matières } \\
\text { impuretés }\end{array}$} & \multirow{3}{*}{ volatiles - } & \multicolumn{2}{|c|}{ - L'eau n'a pas de valeur marchande } \\
\hline & & $\begin{array}{l}\text { - Risque } \\
\text { d'hydrolyse }\end{array}$ & \\
\hline & & \multicolumn{2}{|c|}{ - Démucilagination partielle (fond de bac) } \\
\hline \multicolumn{2}{|c|}{ Acidité oléique } & $\begin{array}{l}\text { - Trop } \\
\text { élevée p }\end{array}$ & $\begin{array}{l}\text { Traceur de mauvaise qualité (hydrolyse des TG, } \\
\text { trouble) baisse des rendements }\end{array}$ \\
\hline \multicolumn{2}{|c|}{ Couleur (T $420 \mathrm{~nm}$ ) } & $\begin{array}{l}\text { - Trop } \\
\text { élevée p }\end{array}$ & $\begin{array}{l}\text { Huile raffinée colorée } \\
\text { Instabilité dans le temps }\end{array}$ \\
\hline Extinctions & $232 \mathrm{~nm}$ & - Trop & Présence de produit d'oxydation (traceur de l'état de \\
\hline
\end{tabular}




\begin{tabular}{|c|c|c|c|}
\hline spécifiques à & $270 \mathrm{~nm}$ & élevée $\quad P$ & dégradation) \\
\hline \multicolumn{2}{|c|}{ Chlorophylle (colza) } & $\begin{array}{l}\text { - Trop } \\
\text { élevée p }\end{array}$ & Traitement particulier $\mathrm{P}$ baisse des rendements \\
\hline \multicolumn{2}{|l|}{ Phosphore } & $\begin{array}{l}\text { - Trop } \\
\text { élevée p }\end{array}$ & Baisse des rendements \\
\hline \multicolumn{2}{|l|}{ Cires } & $\begin{array}{l}\text { - Trop } \\
\text { élevée p }\end{array}$ & $\begin{array}{l}\text { Baisse des } \\
\text { Réduction des cadences }\end{array}$ \\
\hline \multicolumn{2}{|c|}{ Composition en acide gras } & \multicolumn{2}{|c|}{ - Garantie de pureté } \\
\hline \multirow{2}{*}{\multicolumn{2}{|c|}{ Présence de contaminants }} & - Nécessité & de $\quad$ traitements \\
\hline & & \multicolumn{2}{|c|}{ Garantie d'innocuité de l'huile raffinée } \\
\hline
\end{tabular}

Figure 4. Production des huiles brutes : critères à mesurer.

\begin{tabular}{|c|c|c|c|}
\hline $\begin{array}{l}\text { Paramètres } \\
\text { contrôler }\end{array}$ & à Méthode normalisée & Autres possibilités & $\begin{array}{l}\text { Capteurs, } \\
\text { Automates }\end{array}$ \\
\hline Résidu de solvant & $\begin{array}{l}\text { Chromatographie de l'espace } \\
\text { de tête }\end{array}$ & / & / \\
\hline $\begin{array}{l}\text { Impuretés } \\
\text { insolubles }\end{array}$ & Filtration - pesée & / & / \\
\hline Acidité oléique & Titration manuelle & $\begin{array}{l}\text { Réflexion dans le proche } \\
\text { infrarouge } \\
\text { Titrateur automatisé }\end{array}$ & $x$ \\
\hline Phosphore & Colorimétrie & $\begin{array}{l}\text { Absorption } \quad \text { atomique } \\
\text { Spectrométrie d'émission à } \\
\text { plasma }\end{array}$ & 1 \\
\hline Excès de soude & / & Test d'atelier & $x$ \\
\hline Savons & Titration manuelle & Titrateur automatisé & $x$ \\
\hline Matières volatiles & $\begin{array}{l}\text { Dessiccation } \\
\text { Karl fisher }\end{array}$ & (Constante diélectrique) & (turbidimétrie) \\
\hline Cires & / & test & / \\
\hline
\end{tabular}




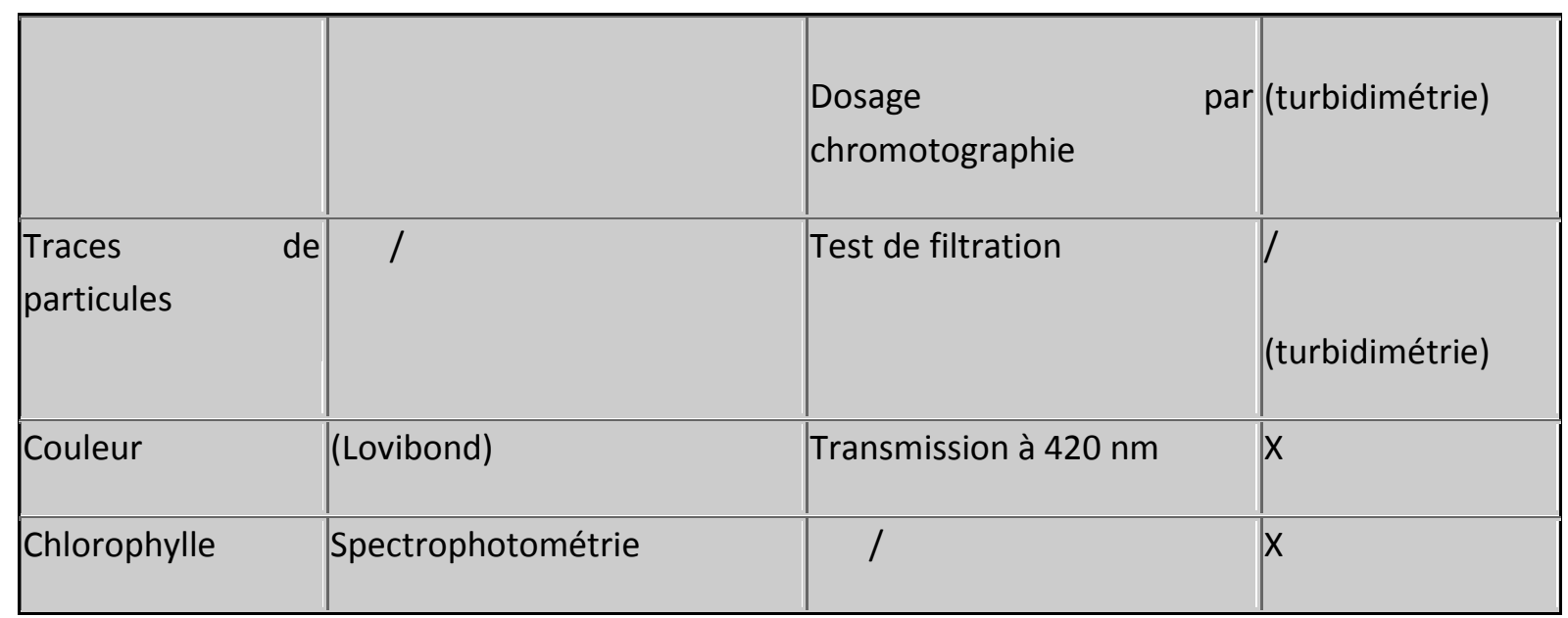

Figure 5. Raffinage chimique des huiles.

\section{Résidu de solvant}

Compte tenu des faibles teneurs à mesurer, ce dosage ne peut être fait qu'en laboratoire.

\section{Impuretés}

C'est là aussi une détermination de laboratoire.

\section{Acidité}

L'acidité est mesurée sur les réservoirs des huiles brutes mises en œuvre et des huiles raffinées mais aussi pour s'assurer de l'efficacité de la neutralisation. II n'est pas nécessaire d'assurer en continu cette mesure puisque l'acidité est supposée rester inchangée dans un réservoir à moins qu'il n'y ait eu des recyclages. Un simple dispositif de titration avec burette permet aux conducteurs de ligne d'avoir l'information en quelques minutes. Les dispositifs classiques de laboratoire par titrage potentiométrique permettent de mesurer avec plus de précision l'acidité, et certains de ces appareils sont automatisés pour être utilisés par des non techniciens, évitant ainsi toute manipulation. Signalons aussi que certains constructeurs d'appareils de mesure de transmission dans le proche infrarouge revendiquent la possibilité de mesurer l'acidité ainsi que le phosphore et l'indice d'iode.

\section{Phosphore}

De l'avis général des responsables de raffinage chimique, c'est un paramètre essentiel à contrôler.

Si la détermination du phosphore doit être faite au départ sur l'huile brute, il est capital de la faire aussi après les différentes étapes du raffinage en particulier sur l'huile neutralisée et séchée. Des teneurs trop élevées après neutralisation $(>25 \mathrm{ppm})$ sont la conséquence de problèmes au prédécirage et au terrage. La méthode normalisée par calcination puis colorimétrie est longue. Les équipements d'absorption atomique avec four graphite ainsi que ceux basés sur la spectrométrie d'émission à plasma permettent de mesurer les faibles teneurs en phosphore. Elles sont, à notre avis, inexploitables pour les huiles brutes, certains résultats étant systématiquement en défaut par rapport à la technique classique de minéralisation par colorimétrie. Malgré tout, ces équipements sont utilisés dans les cellules de contrôle d'atelier car ils permettent d'obtenir la concentration en phosphore en quelques minutes. Un dosage en continu du phosphore sur huile après séchage et décoloration serait malgré tout très utile. 
Un souhait supplémentaire serait de différencier le phosphore minéral du phosphore organique après neutralisation car il est indispensable de s'assurer qu'il n'y ait plus de phosphore minéral au risque d'avoir des phénomènes d'hydrolyse dans l'huile raffinée.

\section{Excès de soude}

Outre le phosphore, un critère très important à suivre par le responsable d'atelier est la mesure de l'excès de soude par rapport à la quantité strictement nécessaire, ceci afin d'éviter la saponification dite parasitaire des triglycérides. Cette mesure doit bien sûr être réalisée très rapidement à proximité de la ligne. Une telle mesure en continu est donc intéressante car elle permet de visualiser des défauts de réglage. Dans les années 85 nous avions construit: un automate de titration qui permettait de suivre en temps réel l'excès de soude par rapport à la quantité nécessaire mais cet appareil n'a finalement pas répondu aux attentes des ateliers de raffinage car il nécessitait beaucoup de maintenance. Nous avons donc mis au point un test colorimétrique rapide qui, réalisé par les opérateurs, leur permet de corriger l'ajout de soude en cas d'excès trop important.

\section{Savons}

La teneur résiduelle en savons est aussi un indicateur indispensable pour le prédécirage et les lavages. La méthode repose encore sur un titrage qui, bien que simple à effectuer, pourrait être automatisé.

\section{Pertes en huile}

Les étapes de neutralisation, prédécirage, lavage étant réalisées par centrifugation, il n'y a pas de méthodes physico-chimiques quantifiant rapidement les pertes en huile neutre. II faut recourir à des tests peu commodes comme la mesure de l'acidité des pâtes de neutralisation et l'examen visuel des eaux de lavage. Ainsi, à défaut d'avoir des méthodes rapides pour doser l'huile neutre perdue à la centrifugation, les opérateurs ont pour seul recours la comparaison des débits massiques de l'huile brute et de l'huile neutralisée.

\section{Absence de limpidité}

Les origines d'un trouble dans une huile raffinée sont les traces d'humidité, la présence de cires et autres lipides cristallisés, les particules de terre de filtration. L'absence d'humidité peut bien sûr être mesurée par des techniques de pertes à l'étuve, discutables, par les titrages avec la méthode de Karl Fisher à l'aide d'appareils volumétriques ou coulométriques dont il existe des formes automatisées fonctionnant sur ligne. Mais la crainte du raffineur est d'avoir des gouttelettes d'eau suite à la fuite d'un échangeur ou d'autre incident. Dans ce cas il n'y a pas réellement de système de détection rapide car les capteurs à constante diélectrique ne sont efficaces que si les gouttelettes d'eau sont de taille et en nombre importants. La mise en évidence d'un défaut de décirage ne peut être faite qu'avec un test au froid qui selon les conditions de temps et de température peut donner des résultats erronés, la tendance de celui qui l'effectue étant de raccourcir le temps de maturation des cristaux pour réduire le délai de réponse au risque que les cristaux ne soient pas suffisamment gros pour être visibles. Sinon il faut recourir au dosage spécifique des cires, qui nécessite un personnel qualifié et un équipement de laboratoire. Ce dosage est cependant indispensable dans le cas du prédécirage, le cold test étant alors inopérant. Une mesure en continu à ce niveau permettrait de mieux gérer l'étape de décirage final (quantité de terre filtrante, capacité de filtration). 
Le passage éventuel de particules peut être détecté rapidement à l'aide d'un test de filtration à proximité de la ligne. Les turbidimètres de ligne permettent de visualiser les gros incidents à l'origine d'une turbidité comme la rupture d'un échangeur au refroidissement à la désodorisation, le passage de particules lors du relargage d'un filtre mais ne garantiront pas toujours l'absence de turbidité de I'huile revenue à température ambiante. Il faut alors recourir à un examen en laboratoire pour évaluer l'origine de la turbidité de l'huile. Pour les mesures de la couleur et des teneurs en chlorophylle, il existe des colorimètres de ligne qui permettent d'automatiser par exemple le poste de terrage. Ils ne peuvent pas se substituer à une mesure effectuée avec un spectrocolorimètre ou un spectrophotomètre de laboratoire mais ils rendent un service appréciable à la conduite de la ligne de raffinage. La plupart de ces contrôles (figure 5) doivent aussi être effectués pour confirmation sur I'huile raffinée après désodorisation. D'autres mesures sont à réaliser sur le produit fini pour qu'il réponde aux attentes du consommateur, en particulier pour les produits de marque (figure 6).

\begin{tabular}{l} 
Aspect du contenant : \\
\hline - Respect de la législation (migration spécifique - migration totale) \\
\hline - Aspect (absence de déformation, de défauts) (tâches, étiquettes...) \\
\hline Aspect du contenu : \\
\hline - Limpidité \\
\hline - Couleur \\
\hline - État de "fraîcheur" du produit : \\
\hline - Absence de défaut organoleptique \\
\hline - Stabilité organoleptique jusqu'à la DLUO \\
\hline - Performances à l'utilisation (mayonnaise, cuisson, friture,...) \\
\hline Garantie d'innocuité : \\
\hline - Absence de contaminants \\
\hline Conformité avec l'étiquetage : \\
\hline - Informations nutritionnelles \\
\hline
\end{tabular}

Figure 6. Les attentes du consommateur en matière d'huile raffinée. 
Ces contrôles sont généralement assurés en temps différé en cellule d'atelier ou plus souvent en laboratoire.

\section{Aspect du contenant}

Sans entrer dans le détail des contrôles spécifiques aux emballages, il faut cependant évoquer les mesures de compatibilité contenant - contenu qui nécessitent des techniques lourdes pour mesurer les migrations globales et spécifiques et dont l'interprétation est discutable. Par contre, le raffineur recherche un moyen rapide d'évaluation du niveau de saturation en azote des huiles afin d'éviter une surconsommation ou un manque qui se traduiront par une déformation des bouteilles et poseront d'importants problèmes au conditionnement et lors de la palettisation.

\section{Aspect du contenu}

- Limpidité - couleur

L'aspect du contenu est évalué à l'aide d'un examen visuel ou avec un colorimètre ou un turbidimètre. Comme pour le contrôle du décirage, la difficulté tient au fait qu'il s'agit de faire apparaître l'origine du manque de transparence rapidement, ce qui n'est pas aisé pour les produits cristallisables, certains pouvant se former lors de la désodorisation.

\section{Qualité du produit}

- État de fraîcheur

L'état d'oxydation est traditionnellement évalué par la mesure de l'indice de peroxydes. Cette mesure n'est pas difficile à pratiquer mais nécessite beaucoup de soins, la preuve en est la difficulté d'avoir une bonne reproductibilité dans les analyses circulaires. Il existe beaucoup d'autres mesures permettant d'évaluer l'état de fraîcheur d'une huile. Le problème repose essentiellement sur l'interprétation que l'on donne à ces indices, le consommateur n'achetant pas un indice de peroxydes ni un indice de para-anisidine mais des qualités fonctionnelles comme le goût, la stabilité de ce goût jusqu'à la DLUO, des garanties de performances à l'utilisation. C'est pourquoi les industriels attachent beaucoup d'importance aux caractéristiques organoleptiques. L'importance de la dégustation est considérable puisque les différents indices de mesure de l'oxydation ne garantissent pas l'absence de flaveurs désagréables; une huile ayant un indice de peroxydes élevé peut ne pas avoir de défauts organoleptiques nets tandis que l'inverse peut se trouver. L'analyse sensorielle requiert une infrastructure spécifique et en dépit du soin apporté, sa reproductibilité est faible. De plus, elle nécessite une organisation assez lourde et entraîne un délai de réponse qui oblige à stocker les produits avant livraison. La mise sur le marché des nez électroniques depuis plusieurs années a permis à plusieurs industriels des corps gras de tester ces appareils dans leur domaine. Pour les huiles à goût (olive - noix), les résultats ont été plutôt négatifs malgré les allégations des constructeurs.

Pour les huiles raffinées, les résultats sont assez décevants bien qu'à terme, il soit probable que cette technique puisse être utilisée en contrôle qualité usine et se substituer aux panels traditionnels, au moins partiellement. Les attentes dans ce domaine sont considérables non seulement pour notre industrie mais pour toute l'industrie alimentaire. 


\section{Garantie d'innocuité du produit}

La recherche de traces de contaminants dans les huiles nécessite des techniques spécifiques et des équipements généralement de haut de gamme. Des laboratoires officiels habitués à ces déterminations assurent les mesures mais l'expérience montre que les résultats sont souvent erronés du fait qu'il n'ont pas nécessairement l'habitude de traiter les matrices corps gras. Les temps de réponse sont longs et ne permettent pas à l'industriel de réagir rapidement. Ainsi pour le dosage des HAP, si l'on souhaite doser la quinzaine de HAP, il faut 2 à 3 jours avec des contraintes tant dans les manipulations que dans l'équipement, alors qu'une méthode qui donnerait le résultat en quelques heures serait très utile pour l'industriel qui, ainsi, pourrait réagir. II en est de même pour la détermination des pesticides. Nous avons nous-mêmes développé à partir de méthodes publiées des méthodes qui permettent en une demi-journée d'avoir un élément de réponse pour ces deux familles de contaminants. Bien sûr, les résultats obtenus ne peuvent rivaliser avec ceux donnés par une méthode officielle de laboratoire tant en sensibilité qu'en précision, mais, ces techniques sont très utiles pour l'industriel qui ne doit pas être systématiquement tributaire de laboratoires extérieurs.

Du fait de l'accroissement de la demande des contrôles de traces de contaminants, il serait avantageux d'avoir des techniques encore plus rapides qui donneraient sinon les teneurs de chaque contaminant, au moins une teneur globale permettant un tri rapide, seuls les échantillons hors normes seraient alors traités par les méthodes officielles.

\section{Conformité avec l'étiquetage}

Les contrôles de pureté et d'informations nutritionnelles consistent d'abord à déterminer la composition en acides gras et en particulier les acides gras essentiels. Les analyses chromatographiques nécessitent malgré tout un délai de réponse assez long qui gêne les industriels ou les utilisateurs d'huiles à la réception des camions. Un progrès pourrait être réalisé en méthylant in situ l'huile et en chromatographiant avec une colonne très rapide les esters méthyliques tout en conservant une parfaite résolution, en particulier en séparant les isomères cis des trans. Les compléments d'information garantissant la pureté des corps gras s'obtiennent par l'analyse de tocophérols, analyse qui peut être faite en trois quarts d'heure, et l'analyse des stérols qui reste délicate à faire et fastidieuse.

\section{Industries utilisant des huiles végétales raffinées}

II n'est pas possible de lister les attentes de toutes les industries utilisant des huiles raffinées pour la fabrication de produits alimentaires divers. Leurs attentes recouvrent globalement celles qui viennent d'être énumérées. Nous nous limiterons à la fabrication de la margarine et produits apparentés.

Cette industrie est aussi confrontée aux contrôles rapides de la nature et de la qualité des matières premières à réception.

Dans certains cas, la réfractométrie permet un tri rapide évitant des erreurs de la manipulation des réservoirs mais généralement, il faut recourir à la détermination de la composition en acides gras. Les autres mesures peroxydes, acidité, se font par les méthodes physico-chimiques classiques, la couleur à l'aide d'un colorimètre, le dosage des acides gras trans par IR ou par chromatographie selon les teneurs. La réalisation de la phase grasse à partir de plusieurs matières grasses nécessite la 
détermination des courbes de fusion. Autrefois effectuée par dilatométrie, la RMN est désormais le standard international pour cette famille de produits. La réponse est malgré tout longue mais essentielle puisque d'elle dépend le déblocage d'une production. Une méthode plus rapide serait un grand progrès. Compte tenu des nombreuses formules réalisées dans une usine, il faut pouvoir contrôler rapidement la teneur en eau des émulsions. Pour cela, on utilise des thermobalances qui donnent un résultat approximatif après 10 à $20 \mathrm{mn}$. Avec les lignes à grande capacité, il serait préférable de recourir à des systèmes de suivi en continu basés sur la réflexion dans le proche infrarouge ou la conductimétrie. Comme pour l'huilerie, les fabricants de margarine ont à faire face au problème de la dégustation du produit fini mais aussi à celui de la recherche des contaminants avec en plus la complication de séparer la phase grasse de l'émulsion, ce qui n'est pas toujours facile compte tenu des performances des émulsifiants.

\section{Conclusions}

Les industriels produisant ou utilisant des corps gras attendent en matière d'analyse physicochimique :

- des moyens leur permettant d'avoir certaines caractéristiques physico-chimiques en cours de process depuis la réception des matières premières jusqu'à la livraison du produit fini sinon en temps réel au moins dans un délai court. Il s'agit alors de privilégier la rapidité de l'information sur la précision de la mesure.

- pour les analyses différées, des techniques plus faciles à mettre en œuvre en laboratoire industriel que celles actuellement en cours dont quelques exemples ont été mentionnés (hexane, cires, stérols, etc.).

- des systèmes de détection des contaminants, simples d'emploi, qui permettent rapidement de trier les échantillons hors normes.

- un ou des moyens d'évaluation de l'état de fraîcheur des huiles sur lesquels tout le monde s'accorde et qui soient suffisamment simples pour être mis en pratique chez le producteur comme chez l'utilisateur.

Ces outils d'analyses spécifiques à la production industrielle ne remettent pas en cause les techniques d'analyses normalisées effectuées dans un laboratoire expert d'analyse interne ou externe.

II n'y a pas concurrence mais complémentarité. 
Illustrations

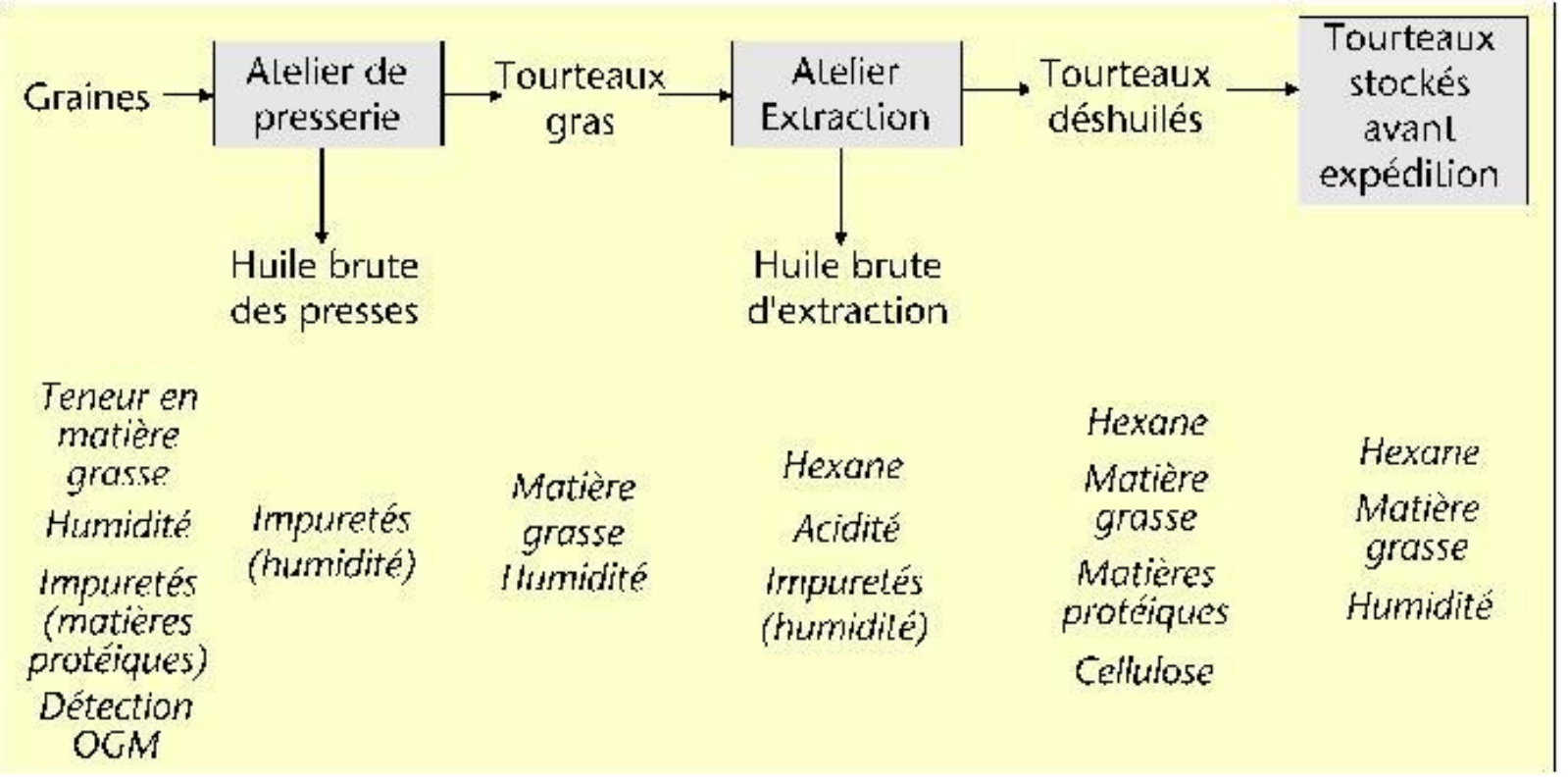

Figure 2. Production des huiles brutes 